\title{
Venous Thromboembolic Complications in Colorectal Surgery
}

\author{
Jung Wook Huh \\ Department of Surgery, Samsung Medical Center, Sungkyunkwan University School of Medicine, Seoul, Korea
}

\section{See Article on Page 187-191}

Colorectal patients who undergo radical surgery are vulnerable to the development of a venous thromboembolism (VTE). A VTE and a pulmonary embolism (PE) can result in chronic leg swelling, ulceration, and discomfort (postthrombotic syndrome), or even death [1]. A number of factors that increase the risk of a postoperative VTE have been identified. In addition to the surgical procedure itself, these include a history of previous VTE, cancer, inflammatory bowel disease, thrombophilia, old age, obesity, smoking, acute illness, wound infection, and prolonged immobilization [2-4]. Therefore, interest exists in better characterizing the specific risk factors for a VET, especially a PE, in patients undergoing colorectal surgery. This could help determine strategies for preventing a VTE, as well as PE, in this complex patient population. Furthermore, questions remain regarding the optimal duration of prophylaxis, patient selection, and the real effect of VTE prophylaxis.

Although multiple trials have demonstrated the efficacy of VTE prophylaxis, a number of reports have also suggested that perioperative VTE prophylaxis is underutilized in clinical practice. The prospective United States VTE Free Study reported that less than one half of the 1,375 patients who had undergone nonorthopedic surgeries and had developed an ultrasound-confirmed VTE had received VTE prophylaxis [5]. In addition, the American College of Chest Physicians Consensus Conference on Antithrombotic Therapy found a VTE prophylaxis compliance of only 13\% [6]. Extended VTE prophylaxis has the potential to negatively influence patient outcomes and may actually lead to bleeding compli-

Correspondence to: Jung Wook Huh, M.D.

Department of Surgery, Samsung Medical Center, Sungkyunkwan University School of Medicine, 81 Irwon-ro, Gangnam-gu, Seoul 06351, Korea

Tel: +82-2-3410-1826, Fax: +82-2-3410-6980

E-mail: jungwook.huh@gmail.com

(C) 2015 The Korean Society of Coloproctology

This is an open-access article distributed under the terms of the Creative Commons Attribution NonCommercial License (http://creativecommons.org/licenses/by-nc/3.0) which permits unrestricted noncommercial use, distribution, and reproduction in any medium, provided the original work is properly cited. cations in the immediate postoperative period. Surgeons may also be more reluctant to perform imaging procedures when the patient's condition is questionable.

Recent prospective data showed the lack of any benefit from an aggressive VTE prevention strategy; among 16,120 patients undergoing colorectal surgery, the use of perioperative and in-hospital VTE prophylaxis increased significantly from $32 \%$ to $86 \%$ and from $60 \%$ to $91 \%$, respectively, between 2006 and 2011 [7]. Despite this increase, no change in the VTE rates was observed over time. The overall incidence of a VTE remained low, at 2.2\%. Another recent study from 2,838 hospitals in the United States indicated that hospitals with characteristics suggestive of higher quality (e.g., more accreditations) had higher VTE chemoprophylaxis rates, but paradoxically had worse risk-adjusted VTE rates [8]. Therefore, VTE rates seem to be influenced more by the utilization rates for hospital VTE imaging than by adherence to VTE prevention strategies.

Based on emerging evidence, further investigations of VTE chemoprophylaxis in patients undergoing colorectal surgery, by using a nation-wide study in Korea, may be necessary. These results may influence future studies looking specifically at prophylaxis guidelines.

\section{CONFLICT OF INTEREST}

No potential conflict of interest relevant to this article was reported.

\section{REFERENCES}

1. Prandoni P, Lensing AW, Cogo A, Cuppini S, Villalta S, Carta M, et al. The long-term clinical course of acute deep venous thrombosis. Ann Intern Med 1996;125:1-7.

2. Monn MF, Hui X, Lau BD, Streiff M, Haut ER, Wick EC, et al. Infection and venous thromboembolism in patients undergoing colorectal surgery: what is the relationship? Dis Colon Rectum 2014;57:497-505.

3. Moghadamyeghaneh Z, Hanna MH, Carmichael JC, Nguyen NT, Stamos MJ. A nationwide analysis of postoperative deep vein 
thrombosis and pulmonary embolism in colon and rectal surgery. J Gastrointest Surg 2014;18:2169-77.

4. Kim J, Bae BN, Jung HS, Park I, Cho H, Gwak G, et al. Risk factors of a pulmonary thromboembolism after colorectal surgery. Ann Coloproctol 2015;31:187-91.

5. Seddighzadeh A, Zurawska U, Shetty R, Goldhaber SZ. Venous thromboembolism in patients undergoing surgery: low rates of prophylaxis and high rates of filter insertion. Thromb Haemost 2007;98:1220-5.
6. Colorectal Writing Group for the Surgical Care and Outcomes Assessment Program-Comparative Effectiveness Research Translation Network (SCOAP-CERTAIN) Collaborative, Nelson DW, Simianu VV, Bastawrous AL, Billingham RP, Fichera A, et al. Thromboembolic complications and prophylaxis patterns in colorectal surgery. JAMA Surg 2015;150:712-20.

7. Bilimoria KY, Chung J, Ju MH, Haut ER, Bentrem DJ, Ko CY, et al. Evaluation of surveillance bias and the validity of the venous thromboembolism quality measure. JAMA 2013;310:1482-9. 